University of Nebraska - Lincoln

DigitalCommons@University of Nebraska - Lincoln

Ralph Skomski Publications

Research Papers in Physics and Astronomy

May 1993

\title{
Crystal field in nitrogenated rare-earth intermetallics
}

\author{
Ralph Skomski \\ University of Nebraska-Lincoln, rskomski2@unl.edu \\ M.D. Kuz'min \\ Trinity College, Dublin, Ireland \\ J.M.D. Coey \\ Trinity College, Dublin, Ireland
}

Follow this and additional works at: https://digitalcommons.unl.edu/physicsskomski

Part of the Physics Commons

Skomski, Ralph; Kuz'min, M.D.; and Coey, J.M.D., "Crystal field in nitrogenated rare-earth intermetallics" (1993). Ralph Skomski Publications. 34.

https://digitalcommons.unl.edu/physicsskomski/34

This Article is brought to you for free and open access by the Research Papers in Physics and Astronomy at DigitalCommons@University of Nebraska - Lincoln. It has been accepted for inclusion in Ralph Skomski Publications by an authorized administrator of DigitalCommons@University of Nebraska - Lincoln. 


\title{
Crystal field in nitrogenated rare-earth intermetallics
}

\author{
R. Skomski, M. D. Kuz'min, and J. M. D. Coey \\ Physics Department, Trinity College, Dublin 2, Ireland
}

The crystal-field in $\mathrm{Sm}_{2} \mathrm{Fe}_{17} \mathrm{~N}_{3-\delta}$ and $\mathrm{Sm}\left(\mathrm{Fe}_{11} \mathrm{Ti}\right) \mathrm{N}_{1-\delta}$ due to interstitial nitrogen has been investigated. Intrinsic parametrization in the superposition model allows separation of the crystal field created by a neighboring nitrogen atom from a purely geometrical factor, which is different for $\mathrm{Sm}_{2} \mathrm{Fe}_{17} \mathrm{~N}_{3-\delta}$ and $\mathrm{Sm}\left(\mathrm{Fe}_{11} \mathrm{Ti}\right) \mathrm{N}_{1-\delta}$. Using published magnetic data, values for the intrinsic parameter $\widetilde{A}_{2}$ per nitrogen atom of $A_{2}=200 \pm 60 \mathrm{Ka} a_{0}^{-2}$ and $\widetilde{A}_{2}=270 \pm 60 \mathrm{Ka}_{0}^{-2}$ for $\mathrm{Sm}_{2} \mathrm{Fe}_{17} \mathrm{~N}_{3-\delta}$ and $\mathrm{Sm}\left(\mathrm{Fe}_{11} \mathrm{Ti}\right) \mathrm{N}_{1-\delta}$, respectively, are obtained. Because of charge penetration, which is discussed in the form of an explicit crystal-field weight function, it is not possible to interpret $A_{2}^{0}$ or $\widetilde{A}_{2}$ as crystal-field parameters independent of the $4 f$ ion.

\section{INTRODUCTION}

The discovery that nitrogen or carbon absorption drastically improves the magnetic properties of $\mathrm{Sm}_{2} \mathrm{Fe}_{17}$ has sparked interest in the gas-phase interstitial modification of rare-earth intermetallics. Interstitial modification leads to a volume expansion of about $6 \%$, which is the main cause for the improvement of saturation magnetization and Curie temperature of $\mathrm{Sm}_{2} \mathrm{Fe}_{17}$ (see Refs. 1 and 2). However, the change from easy-plane to strong easy-axis anisotropy is ascribed to the modification of the rare-earth crystal field due to the surrounding nitrogen or carbon atoms. ${ }^{1,2}$ Another example of interstitial modification of a rare-earth intermetallic is easy axis $\mathrm{Sm}\left(\mathrm{Fe}_{11} \mathrm{Ti}\right)$, whose nitride $\mathrm{Sm}\left(\mathrm{Fe}_{11} \mathrm{Ti}\right) \mathrm{N}_{\mathrm{I}-\delta}$ shows easy-plane anisotropy. ${ }^{3}$

Interstitial nitrogen atoms in $\mathrm{Sm}_{2} \mathrm{Fe}_{17} \mathrm{~N}_{3-\delta}$ and $\mathrm{Sm}\left(\mathrm{Fe}_{11} \mathrm{Ti}\right) \mathrm{N}_{1-\delta}$ occupy the large octahedral $9 e$ sites in the $\mathrm{Th}_{2} \mathrm{Zn}_{17}$ structure and $2 b$ sites in the $\mathrm{ThMn}_{12}$ structure nearly exclusively. ${ }^{2,3}$ The coordination of rare-earth atoms by neighboring interstitials is different in the two structures; the $9 e$ sites form an in-plane triangle, whereas the $2 b$ sites form an axial dumbell (Fig. 1). This provides a qualitative explanation of the observed anisotropy trends, if we take into account the electrostatic repulsion between the prolate $\mathrm{Sm}^{3+} 4 f$ shell and negatively charged interstitial atoms.

Here, we use the intrinsic parametrization of the superposition model ${ }^{4}$ to separate the effect of the geometric arrangement of the nitrogen atoms (Fig. 1) from the underlying strength of the crystal field created by a single nitrogen atom.

\section{MODEL AND CALCULATION}

The following assumptions are made.

(i) The compound consists of two sublattices: a ferromagnetic $\mathrm{Fe}$ sublattice with the magnetization $\mathbf{M}_{\mathrm{Fe}}$ and a paramagnetic $\mathrm{Sm}$ sublattice exposed to the $\mathrm{Sm}-\mathrm{Fe}$ exchange field $\mathbf{B}_{\mathrm{ex}}=n_{\mathrm{Sm}-\mathrm{Fe}} \mu_{0} \mathbf{M}_{\mathrm{Fe}}$. The Sm-Sm exchange is neglected, while the $\mathrm{Sm}-\mathrm{Fe}$ exchange is assumed to be isotropic. The $\mathrm{Sm}$ anisotropy is believed to originate from the electrostatic crystal field acting on the $4 f$ shells of the $\mathrm{Sm}^{3+}$ ions in their $J=5 / 2$ ground state; $J$-mixing effects are neglected. The crystal-field interaction is treated as a perturbation with respect to the $\mathrm{Sm}-\mathrm{Fe}$ exchange, so the unperturbed $4 f$ wave functions can be used to calculate the crystal-field energy.

(ii) At room temperature, only the lowest-order crystal-field interaction parameter $A_{2}^{0}$ is taken into account. ${ }^{5}$ To describe the rare-earth crystal-field interaction we use the notation ${ }^{6}$

$$
\mathbb{H}_{\mathrm{CF}}=\alpha_{J} \mathbb{1}_{2}^{0}\left\langle r^{2}\right\rangle O_{2}^{0}
$$

The temperature dependence of the Stevens operator expressions has been treated by Kuz'min who finds

$$
\left\langle O_{2}^{0}\right\rangle=\frac{25}{2} B^{2}{ }_{5 / 2}\left(\frac{25}{7} \frac{n_{\mathrm{R}-\mathrm{Fe}} \mu_{0} \mathbf{M}_{\mathrm{Fe}}}{k T}\right)
$$

and

$$
K_{1}=K_{\mathrm{Fe}}-\frac{65}{84} A_{2}^{0}\left\langle r^{2}\right\rangle B^{2}{ }_{5 / 2}\left(\frac{25}{7} \frac{n_{\mathrm{R}-\mathrm{Fe}} \mu_{0} \mathbf{M}_{\mathrm{Fe}}}{k T}\right),
$$

where $B_{5 / 2}^{2}(x)$ is the second-order generalized Brillouin function. ${ }^{7}$ As can be seen from Fig. 2, the generalized Brillouin function yields a low-temperature plateau (solid line), whereas the classical Bessel function (dashed lines) fails to match the two observed data points. ${ }^{8,9}$

(iii) The crystal field is supposed to be the sum of independent contributions from all the nearest nitrogen neighbors. ${ }^{4}$ To separate explicitly the purely geometrical effect, we introduce the intrinsic parameter $\widetilde{A}_{2}$, which describes the crystal-field change per nitrogen atom

$$
A_{2}^{0}(c)-A_{2}^{0}(0)+\frac{1}{2} \cos \left(3 \cos ^{2} \theta-1\right) \tilde{A}_{2} \text {. }
$$

Here, $c$ is the nitrogen concentration on the interstitial sites, $\zeta$ the number of interstitial next neighbors, and $\theta$ the

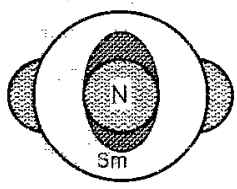

(a)

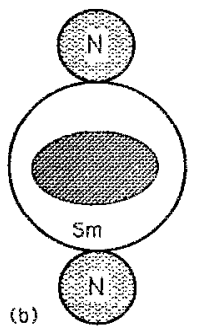

FIG. 1. The coordination of interstitial nitrogen in (a) $\mathrm{Sm}_{2} \mathrm{Fe}_{17} \mathrm{~N}_{3 \ldots s}$ $(\theta=\pi / 2)$ and (b) $\operatorname{Sm}\left(\mathrm{Fe}_{11} \mathrm{Ti}\right) \mathrm{N}_{1 \ldots \delta}(\theta=0)$. 


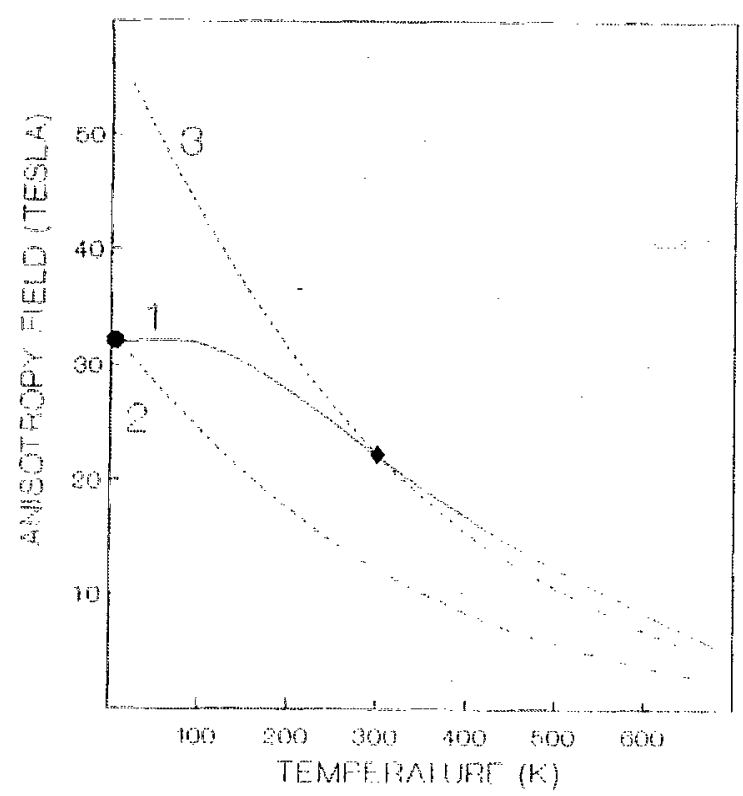

FIG. 2. Temperature dependence of the anisotropy field of $\mathrm{Sm}_{2} \mathrm{Fe}_{17} \mathrm{~N}_{3--\delta}$. Solid line (curve 1): calculated using Eq. (2) with,$A_{2}^{0}=-340 \mathrm{Ka}_{0}^{-2}$. Dashed lines: calculated using the classical Bessel function $\hat{I}_{5 / 2}(x)$ instead of $B^{2}{ }_{5 / 2}(x)$ in Eq. (2) with $A_{2}^{0}=-280 \mathrm{Ka} a_{i j}^{-2}$ (curve 2) and $A_{2}^{0}=-480$ $\mathrm{K} a_{0 \mathrm{j}}{ }^{-2}$ (curve 3). Experimental data: Ref. 7 (circle) and Ref. 8 (square).

corresponding coordination angle, $\theta\left[\mathrm{Sm}\left(\mathrm{Fe}_{11} \mathrm{Ti}\right)\right]=0$ and $\theta\left(\mathrm{Sm}_{2} \mathrm{Fe}_{17}\right)=\pi / 2$ (Fig. 1). To deduce $\widetilde{A}_{2}$ from Eq. (4), we have to compare $A_{2}^{0}(c)$ for at least two different nitrogen concentrations, $c$ and 0 . With the anisotropy field $H_{a}$ $=2 K_{1} / \mu_{0} M_{0}$, we obtain

$$
\tilde{A}_{2}=-\frac{2 \mu_{0}\left[\left(M_{0} H_{a} / v_{R}\right)_{c}-\left(M_{0} H_{a} / v_{R}\right)_{0}\right]}{3 \alpha_{J}\left(3 \cos ^{2} \theta-1\right) c \zeta\left\langle r^{2}\right\rangle\left\langle O_{2}^{0}(c)\right\rangle}
$$

where $v_{\mathrm{R}}$ denotes the density $N_{\mathrm{R}} / V$ of rare-earth atoms.

To determine $\left\langle O_{2}^{0}\right\rangle$ the molecular field constants $n_{\mathrm{R}-\mathrm{Fe}}$ must be known: $n_{\mathrm{R}-\mathrm{Fe}}\left(\mathrm{R}_{2} \mathrm{Fe}_{17}\right)=300 \quad \mu_{0}$ and $n_{\mathrm{R}-\mathrm{Fe}}\left(\mathrm{RFe}_{11} \mathrm{Ti}\right)=340 \mu_{0}$ (see Refs. 10 and 11 ). We will make use of the value $A_{2}^{0}(3 c=2.80 \pm 0.15)=-242 \mathrm{~K} / a_{0}^{2}$ deduced from the room-temperature anisotropy field $\mu_{0} H_{u}$ $=22 \mathrm{~T}$. Similiar considerations yield $A_{2}^{0}(c=0)=35 \mathrm{~K} / a_{0}^{2}$ for the parent compound $\operatorname{Sm}_{2} \mathrm{Fe}_{17}\left(\mu_{0} H_{a}=-4 \mathrm{~T}\right.$ ) (see Ref. 9). The values used for $\mathrm{Sm}\left(\mathrm{Fe}_{11} \mathrm{Ti}\right)$ are $A_{2}^{0}(c=0)$ $\equiv-135 \mathrm{~K} / a_{0}^{2}$ and $A_{2}^{0}(c=0.8)=292 \mathrm{~K} / a_{0}^{2}$ (cf. Ref. 3 and 9). Finally, we obtain for the intrinsic crystal-field per nitrogen atom

$$
\begin{array}{ll}
\widetilde{A}_{2}=+200 \pm 60 \mathrm{~K} a_{0}^{-2}, & \operatorname{Sm}_{2} \mathrm{Fe}_{17} \mathrm{~N}_{3-\delta}, \\
\tilde{A}_{2}=+270 \pm 60 \mathrm{Ka} a_{0}^{-2}, & \operatorname{Sm}\left(\mathrm{Fe}_{11} \mathrm{Ti}\right) \mathrm{N}_{1-\delta},
\end{array}
$$

where the estimated error includes the uncertainty in $c, H_{a}^{*}$, and $\left\langle O_{2}^{\natural}\right\rangle$.

\section{DISCUSSION}

\section{A. Sign and magnitude of $\widehat{\boldsymbol{A}}_{2}$}

The intrinsic parameters Eq. (6) have the same sign and similiar numerical values, although the corresponding anisotropy field values are completely different. This con- firms the idea that the crystal-field modification in $\mathrm{Sm}_{2} \mathrm{Fe}_{17} \mathrm{~N}_{3-\delta}$ and $\mathrm{Sm}\left(\mathrm{Fe}_{11} \mathrm{Ti}\right) \mathrm{N}_{1-\delta}$ is directly due to neighboring nitrogen atoms. Note that electrostatic charges are strongly screened in metals, as opposed to nonmetallic rare-earth compounds, where the lattice summation is much more difficult (cf. Ref. 4).

It is interesting to compare the present results with data available from literature. Equation (4) predicts a linear increase of $A_{2}^{0}$ with the interstitial concentration $c$. Mössbauer and magnetic measurements on $\mathrm{Sm}_{2} \mathrm{Fe}_{17}$ carbides and nitrides indicate a concentration dependence which is, at least approximately, linear, ${ }^{9,12,13}$ but the quantitative situation is less satisfactory. From Ref. 12, an intrinsic crystal field of $\widetilde{A}_{2}=+126 \mathrm{~K} a_{0}^{-2}$ can be deduced for $\mathrm{Sm}_{2} \mathrm{Fe}_{17}$ nitride and carbide, but extrapolation of the experimental data yields $A_{2}^{0}\left(\mathrm{Sm}_{2} \mathrm{Fe}_{17} \mathrm{~N}_{y \rightarrow 0}\right) \approx-50 \mathrm{~K} a_{0}^{-2}$ and $A_{2}^{0}\left(\mathrm{Sm}_{2} \mathrm{Fe}_{17} \mathrm{C}_{y \rightarrow 0}\right) \approx 0$. A possible reason for this inconsistency are inexact concentration values $c$.

It is difficult to decide whether the difference between the two values (6a) and (6b) is significant. Taking into account the distance dependence of of the crystal field $\left(\widetilde{A}_{2} \propto 1 / \mathrm{R}^{3}\right.$ in the point-charge model $)$ and the slightly smaller samarium-nitrogen distance in the 1-12 lattice (5\%), we should expect a difference of about $30 \mathrm{Ka} a_{0}^{-2}$.

\section{B. Crystal-field interaction}

The electrostatic crystal-field energy, which includes charge penetration, is given by

$$
H_{\mathrm{CF}}=\frac{1}{4 \pi \epsilon_{0}} \int \frac{\rho(\mathbf{r}) \rho_{4 f^{\prime}}\left(\mathbf{r}^{\prime}\right)}{\left|\mathbf{r}-\mathbf{r}^{\prime}\right|} d \mathbf{r} d \mathbf{r}^{\prime},
$$

where $\rho_{4 f}(\mathbf{r})$ and $\rho(\mathbf{r})$ are the local $4 f$ density and the density of the non- $4 f$ electrons, respectively. Like any other functions, $\rho_{4 f}(\mathbf{r})$ and $1 /\left|\mathbf{r}-\mathbf{r}^{\prime}\right|$ can be expanded in spherical harmonics. The result is a sum of six-fold integrals, each of them yielding a crystal-field parameter. If $\rho(\mathbf{r})$ is known, e.g., from band structure calculations, these integrals can be solved numerically. To obtain a more physical interpretation of the crystal-field integrals, we represent $A_{2}^{0}$ as a three-fold integral

$$
A_{2}^{0}=-\int\left(3 \cos ^{2} \theta-1\right) W_{2}(r) \rho(\mathbf{r}) d \mathbf{r}
$$

where the crystal-field weight function

$$
W_{2}(r)=\frac{e}{4 \pi \epsilon_{0}} \frac{1}{4\left\langle r^{2}\right\rangle}\left(\int_{0}^{r} \frac{\xi^{4}}{r^{3}} F(\xi) d \xi+\int_{r}^{\infty} \frac{r^{2}}{\xi} F(\xi) d \xi\right)
$$

is characteristic of a given rare-earth metal. Therefore $A_{2}^{0}$ explicitly depends on the $4 f$ charge distribution. Figure 3 shows the function $W_{2}(r)$ for samarium, derived from Hartree-Fock $4 f$ charge density values. ${ }^{14}$ If the source of the crystal field is far away from the rare-earth ion, the $1 / r^{3}$ dependence of the point-charge model is reproduced. For distances below $0.5 \AA$ the crystal-field interaction is much less than expected from the point-charge model.

In the case of point-charge like non- $4 f$ charge clouds the radial dependence of the crystal field is given by Fig. 3 


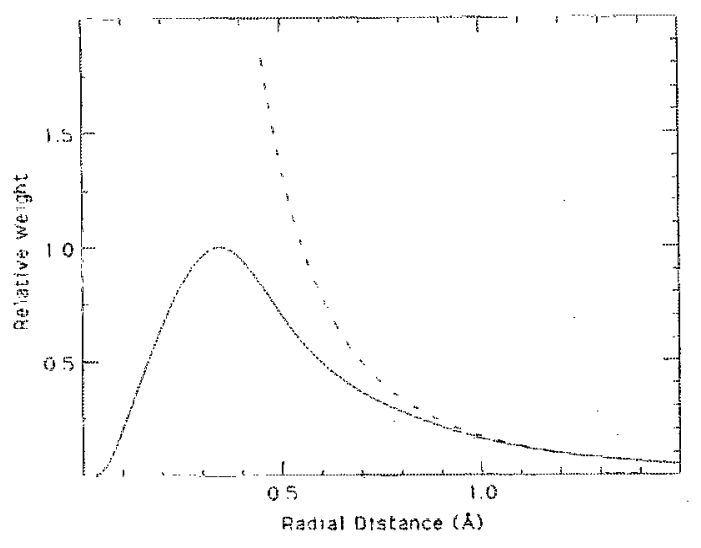

FIG. 3. Radial weight function $W_{2}(r)$ for the second-order crystal-field interaction.

(solid line). Assuming an effective point charge $q_{0}=-3 e$ per nitrogen atom and a Sm-N distance of about $2.45 \AA$, we obtain $\tilde{A}_{2}=4500 \mathrm{Ka} a_{0}^{-2}$. In reality, this large value is reduced by screening and polarization effects to about $5 \%$ of this value.

In Ref. 12, the crystal-field modification is ascribed to fractional bonds ${ }^{15}$ and fictious charge transfer from nitrogen to samarium. With a nitrogen single-bond distance of $d_{N}(1)=0.55 \AA$, the authors were able to achieve excellent agreement between measured and calculated $A_{2}^{0}$ values for $\mathrm{Sm}_{2} \mathrm{Fe}_{17} \mathrm{~N}_{3-\delta}$, but the more realistic value $d_{\mathrm{N}}(1)=0.74$ $\AA^{15}$ yields a factor 2.1 .

Coehoorn et al. investigated the crystal-field contribution of the $5 d$ and $6 p$ electrons and found considerable deviations from the point-charge model behavior. ${ }^{16,17}$ Figure 3 gives an illustrative interpretation of this behavior: due to the strong overlap between valence and $4 f$ electrons, ${ }^{14}$ a part of the valence electrons can be found at $r<0.5 \AA$ where the point-charge model fails. The observed anisotropy trends are explained by the continuity of the electron density at the Wigner-Seitz cell boundary, as opposed to Ref. 12, where the electronegativity difference is the main factor.

\section{CONCLUSIONS}

The intrinsic crystal field contribution per nitrogen atom (ca. $200 \mathrm{Ka}_{0}^{-2}$ ) is comparable in $\mathrm{Sm}_{2} \mathrm{Fe}_{17} \mathrm{~N}_{3-\delta}$ and $\mathrm{Sm}\left(\mathrm{Fe}_{11} \mathrm{Ti}\right) \mathrm{N}_{1 \ldots \delta}$, which indicates that the nitrogen provides a common mechanism of crystal-field modification in both compounds. A crystal-field weight function is introduced to show that charge penetration is negligible for a localized interstitial, but is important as far as polarization of non- $4 f$ orbitals is concerned.

\section{ACKNOWLEDGMENT}

This work was supported by the BRITE/EURAM programme of the European Commission. It forms part of the Concerted European Action on Magnets.

'J. M. D. Coey and H. Sun, J. Magn. Magn. Mater. 87, L251 (1990).

${ }^{2}$ H. Sun, J. M. D. Coey, Y. Otani, and D. P. F. Hurley, J. Phys.: Condens. Matter 2, 6465 (1990).

${ }^{3}$ J. M. D. Coey, Physica Scripta T 39, 21 (1991).

${ }^{4}$ D. J. Newman and B. Ng, Rep. Prog. Phys. 52, 699 (1989).

${ }^{3}$ This is justified by the low $\beta_{J}$ and zero $\gamma_{J}$ values of the $J=5 / 2$ ground state of $\mathrm{Sm}^{3+}$, by the pronounced distance and temperature dependences of the higher-order crystal-field interaction, and by the fact that the fourth-order coordination factor ${ }^{4}$ has the same sign for $\theta=\pi / 2$ and $\theta=0$.

${ }^{6}$ M. T. Hutchings, Solid State Phys. 16, 227 (1964).

${ }^{7}$ M. D. Kuz'min, Phys. Rev. B 46, 8219 (1992).

${ }^{8}$ Y. Lu, O. Tegus, Q. A. Li, N. Tang, M. J. Yu, R. W. Zhao, J. P. Kuang, F. M. Yang, G. F. Zhou, X. Li, and F. R. de Boer, Physica B 177, 243 (1992).

${ }^{9}$ M. Katter, J. Wecker, C. Kuhrt, L. Schultz, and R. Grössinger, J. Magn. Magn. Mater. 117, 419 (1992).

${ }^{10}$ J. M. D. Coey, H. Sun, and D. P. F. Hurley, J. Magn. Magn. Mater. 101, 310 (1991).

${ }^{11}$ H.S. Li and J. M. D. Cuey, J. Magin. Magn. Mater, 115, 152 (1992).

${ }^{12} \mathrm{H} .-\mathrm{S}$. Li and J, M. Cadogan, Proceedings of the 7 th International Symposium on Magnetic Anisotropy and Coercivity in RE-TM Alloys, Canberra, Australia, July 1992 (University of Western Australia, Perth), p. 185.

${ }^{13}$ K. H. J. Buschow, Rep. Prog. Phys. 54, 1123 (1991).

${ }^{14}$ A. J. Freeman and R. E. Watson, Phys. Rev. 127, 2058 (1962).

${ }^{15} \mathrm{~L}$. Pauling, The Nature of the Chemical Bond (Cornell U.P., New York, 1960).

${ }^{16}$ M. W. Dirken, R. C. Thiel, R. Coehoorn, T. H. Jacobs, and K. H. J. Buschow, J. Magn. Magn. Mater. 94, L15 (1991).

${ }^{17}$ R. Coehoorn. K. H. J, Buschow, M. W. Dirken, and R. C. Thiel, Phys. Rev. B 42, 4645 (1990). 